\title{
Special rank one groups are perfect
}

page $1 / 3$

go back

full screen

close

quit

\author{
Anja Steinbach
}

Abstract

We give a new proof of the fact that special (abstract) rank one groups with arbitrary unipotent subgroups of size at least 4 are perfect.

Keywords: special (abstract) rank one group, Moufang set

MSC 2000: Primary 20E42; Secondary 51E42

\section{Introduction}

J. Tits [3] defined Moufang sets in order to axiomatize the linear algebraic groups of relative rank one. A closely related concept of so-called (abstract) rank one groups has been introduced by F. G. Timmesfeld [2].

Here a group $X$ is an (abstract) rank one group with unipotent subgroups $A$ and $B$, if $X=\langle A, B\rangle$ with $A$ and $B$ different subgroups of $X$, and (writing $\left.A^{b}=b^{-1} A b\right)$

for each $1 \neq a \in A$, there is an element $1 \neq b \in B$ such that $A^{b}=B^{a}$, and vice versa.

We emphasize that in contrast to Timmesfeld's definition [2, p. 1] we do not assume that $A$ and $B$ are nilpotent. In an (abstract) rank one group $X$ with unipotent subgroups $A$ and $B$, the element $1 \neq b \in B$ with $A^{b}=B^{a}$ is uniquely determined for each $1 \neq a \in A$ (as $A \neq B$ ) and denoted by $b(a)$. Similary we define $a(b)$.

We say $X$ is special, if $b\left(a^{-1}\right)=b(a)^{-1}$ for all $1 \neq a \in A$. This is equivalent with Timmesfeld's original definition, see Timmesfeld [2, I (2.2), p. 17]. In this note, we give a new proof of the following result.

Theorem 1.1. Any special (abstract) rank one group with arbitrary unipotent subgroups of size at least 4 is perfect. 

When $A \cap X^{\prime}=1$, then Lemma 2.2 implies that $A \subseteq\left\{1, a, a^{-1}\right\}$, where $1 \neq a \in A$; i.e., $|A| \leq 3$. Thus for $|A| \geq 4$, we may choose $1 \neq a \in A \cap X^{\prime}$. By Lemma 2.2, we obtain $A \subseteq a\left(A \cap X^{\prime}\right) \cup\left\{1, a^{-1}\right\} \subseteq A \cap X^{\prime}$, as desired.

\section{References}

[1] T. De Medts, Y. Segev and K. Tent, Special Moufang sets, their root groups and their $\mu$-maps, Proc. Lond. Math. Soc. 96 (2008), no. 3, 767-791.

[2] F. G. Timmesfeld, Abstract root subgroups and simple groups of Lie type, Monographs in Mathematics 95, Birkhäuser, 2001.

[3] J. Tits, Twin buildings and groups of Kac-Moody type, in Groups, combinatorics \& geometry (Durham, 1990), 249-286, London Math. Soc. Lecture Note Ser. 165, Cambridge Univ. Press, Cambridge, 1992.

Anja Steinbach

Justus-Liebig-Universität Giessen, Mathematisches Institut, Arndtstrasse 2, D 35392 GIESSEN, GERMANY

e-mail: Anja.Steinbach@math.uni-giessen.de 